\title{
High source potential upstream of a current-free electric double layer
}

\author{
C. Charles ${ }^{\text {a) }}$ \\ Plasma Research Laboratory, Research School of Physical Sciences and Engineering, \\ The Australian National University, Acton, Australian Capital Territory 0200, Australia
}

(Received 17 November 2004; accepted 4 February 2005; published online 1 April 2005)

\begin{abstract}
Large plasma potentials (up to $\sim 80 \mathrm{~V}$ ) are measured upstream of a current-free electric double layer generated in a helicon rf argon discharge for a constant rf power of $250 \mathrm{~W}$ (at $13.56 \mathrm{MHz}$ ) and for low operating pressures $(<2$ mTorr $)$ using an electrostatic ion energy analyzer. The energy of the $\mathrm{Ar}^{+}$ion beam formed by acceleration through the potential drop of the double layer is found to be strongly correlated with the large source potentials: both the source potential and the beam energy increase with decreasing pressure. The creation of the double layer depends on the magnetic field generated by the solenoid near the closed end (glass plate) of the helicon source. (C) 2005 American Institute of Physics. [DOI: 10.1063/1.1883182]
\end{abstract}

Electric double layers (DLs) occur naturally in a variety of space plasma environments ${ }^{1,2}$ and are being characterized using satellites. ${ }^{3}$ Terrestrial laboratory experiments have been successfully demonstrated for decades in the case of current-driven DLs (Refs. 4,5) and more recently of currentfree (but driven by an applied potential) propagating or stationary DLs. ${ }^{6,7}$ Current-driven DLs generated by a constriction in a gas-discharge tube ${ }^{8}$ or by plasma expansion in a diverging magnetic field ${ }^{9}$ are well documented. During the past few years, current-free DLs have been measured in the laboratory in the context of a low pressure helicon plasma expansion in a diverging magnetic field. ${ }^{10-13}$ Nakano et al. ${ }^{14}$ also proposed a double layer as a possible acceleration process responsible for large ion flows measured using laser induced fluorescence in a low pressure chlorine/helium plasma expanding from an electron cyclotron resonance source.

Although most helicon experiments show DLs which are apparently current free, virtually all interpretations of space measurements have described the existence of currents, which are assumed to be the energy source of the potential drop of the double layer. As a consequence, most theoretical ${ }^{15}$ and computer simulation ${ }^{16}$ works have also dealt with current-driven DLs. Similarly, in terrestrial laboratory experiments, a potential difference is imposed on two separately created plasmas which are allowed to interact, and, under certain conditions, lead to DLs. Generally, these initial experimental conditions lead to a current flow through the plasma, although this is not a necessary and sufficient condition for the existence of the double layer. ${ }^{17}$ Nevertheless, either the potential or the current has to be imposed as an initial condition. For the present experiments, neither of these parameters are specified.

In this paper, the plasma potentials upstream and downstream of a current-free electric double layer as well as the double-layer potential drop are measured as a function of gas pressure with a magnetic field of about $135 \mathrm{G}$ in the source and a rf power of $250 \mathrm{~W}$. The creation of the DL is also

${ }^{a)}$ Electronic mail: christine.charles@anu.edu.au investigated when reducing the magnetic field generated by the solenoid near the closed end (glass plate) of the helicon source and when changing the position of the glass plate terminating the source.

A previously described ${ }^{12}$ horizontal helicon system consisting of a $15 \mathrm{~cm}$ diameter helicon source $(31 \mathrm{~cm}$ long cylindrical glass tube terminated with a $0.5 \mathrm{~cm}$ thick glass plate and surrounded by an $18 \mathrm{~cm}$ long double-saddle antenna) is attached contiguously to a $30 \mathrm{~cm}$ long $32 \mathrm{~cm}$ diam earthed aluminum diffusion chamber. The antenna is fed from a rf matching network/generator system operating at $13.56 \mathrm{MHz}$. For all experiments, the rf power was kept constant at $250 \mathrm{~W}$. The argon feed gas is introduced on the side of the chamber, and a turbomolecular/rotary pumping system is connected to the sidewall of the chamber. The base pressure is $\sim 2$ $\times 10^{-6}$ Torr, the pressure being measured with an ion gauge and a baratron gauge.

Previous experiments in the same system showed the presence of a current-free DL at $z=25 \mathrm{~cm}$ for an argon discharge ${ }^{10}$ where $z=0 \mathrm{~cm}$ is defined at the closed end of the helicon source. More recently an $\mathrm{Ar}^{+}$ion beam has been detected arising from the DL using a gridded energy analyser. ${ }^{12}$ These previous experiments quoted a current of 6 A through each of the magnetic field coils surrounding the source tube and a subsequent calculated field $B_{z}$ of about 250 $\mathrm{G}$ in the source. Recent inspection has shown that each magnetic coil comprises two separate windings in parallel resulting in only 3 A passing through each winding. Consequently the field claimed in our previous publications ${ }^{10,12}$ was overestimated by a factor of 2 . The present experiments were initially conducted under the same initial current conditions and the corresponding measured magnetic field is shown by a solid line on Fig. 1. Subsequently, the current in the solenoid near the closed end of the source is decreased to 5, 4, and $2 \mathrm{~A}$ and the measured field is shown on Fig. 1, respectively. A no field case is also investigated. A second glass plate can be inserted in the source from the left via a port and a hole centered in the stationary glass plate and moved from $z=0$ to $16 \mathrm{~cm}$.

A movable retarding field energy analyzer (RFEA) situ- 


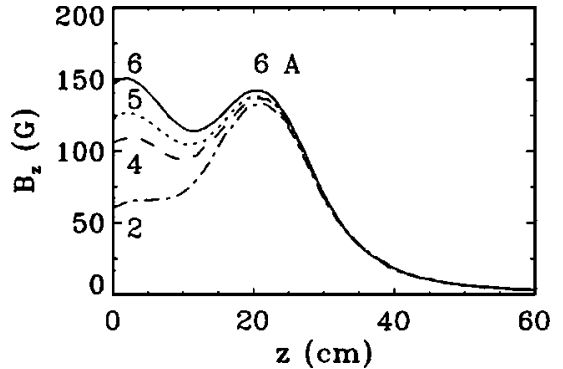

FIG. 1. $B_{z}$ component of the dc magnetic field measured along axis for a constant solenoid current near the source exit (6 A) and a varying solenoid current near the closed end of the source: $6 \mathrm{~A}$ (solid line), $5 \mathrm{~A}$ (dotted line), 4 A (dashed line), and $2 \mathrm{~A}$ (dotted-dashed line).

ated on the $z$ axis of the system, and inserted from the back chamber plate, is initially used to measure the currentdiscriminator voltage characteristics $I\left(V_{d}\right)$ in the source $(z$ $=15 \mathrm{~cm}$ ) with its orifice facing radially. Subsequently it is inserted using a side port on the diffusion chamber and positioned on the $z$ axis $(z=37 \mathrm{~cm})$ with its orifice facing axially, i.e., facing the double layer. A detailed description and optimization of the analyzer have been previously reported. ${ }^{18}$ It showed that the energy resolution is expected to be less than $1 \mathrm{eV}$. The derivative of the collector current versus discriminator voltage $I\left(V_{d}\right)$ characteristics is called the ion energy distribution function (IEDF). For the low density conditions presently investigated $\left(\leqslant\right.$ a few $\left.10^{10} \mathrm{~cm}^{-3}\right)$ no peak separation due to a possible rf oscillation of the sheath is expected. ${ }^{18}$ This is experimentally confirmed by the measurement of single peaked IEDFs around the local plasma potential when the RFEA's orifice is facing radially, i.e., facing the reactor's sidewalls. When the RFEA's orifice is facing the DL, a second peak at higher energy is detected which corresponds to the beam resulting from ion acceleration in the potential drop of the double layer.

The $I\left(V_{d}\right)$ characteristic is initially measured using the RFEA positioned in the center of the source at $z=15 \mathrm{~cm}$ as a function of decreasing pressure starting at 3 mTorr as one continuous sweep for a rf power of $250 \mathrm{~W}$ and for the maximum magnetic field configuration shown by a solid line on Fig. 1. All corresponding IEDF's exhibit a single peak distribution around the plasma potential in the source center $V_{\text {source }}$. The source potential versus pressure is shown by crosses in Fig. 2. It decreases quite rapidly with increasing pressure. At 0.3 mTorr, $V_{\text {source }}$ is $58.6 \mathrm{~V}$.

The $I\left(V_{d}\right)$ characteristic is subsequently measured with the RFEA positioned on the $z$ axis $(z=37 \mathrm{~cm})$ in the diffusion chamber but facing the source, i.e., the DL, for a rf power of $250 \mathrm{~W}$ and for the same magnetic field configuration shown by a solid line on Fig. 1. The double layer is initially generated at a pressure of about 0.3 mTorr and the measurements are taken in a continuous pressure sweep increasing the pressure to about 1.5 mTorr. All corresponding IEDF's show the presence of two peaks: analysis of such bimodal IEDFs measured with the RFEA located $12 \mathrm{~cm}$ downstream of the DL and facing the DL has been previously described in detail ${ }^{12}$ and results from the detection of a population of cold ions located near the position of the

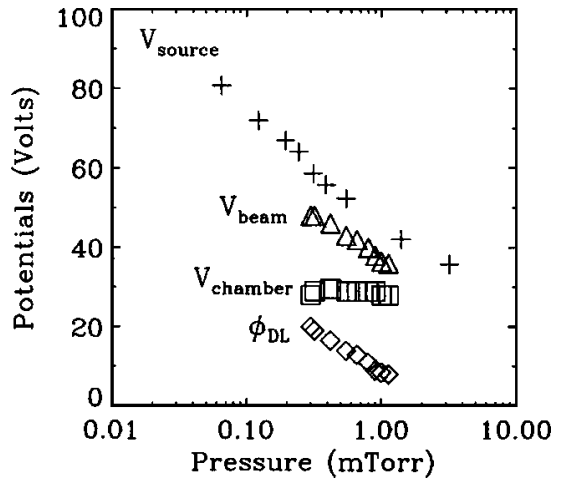

FIG. 2. Plasma potential in the source $V_{\text {source }}($ crosses, $z=15 \mathrm{~cm}$ ), ion beam energy $V_{\text {beam }}$ (triangles, $z=37 \mathrm{~cm}$ ), plasma potential in the chamber $V_{\text {chamber }}$ (squares, $z=37 \mathrm{~cm}$ ), and double-layer potential drop $\phi_{D L}$ (diamonds, $z$ $=37 \mathrm{~cm}$ ) as a function of gas pressure for $250 \mathrm{~W}$ rf power and the maximum field condition shown by a solid line on Fig. 1.

RFEA (low energy peak corresponding to the local plasma potential in the chamber $V_{\text {chamber }}$ at $z=37 \mathrm{~cm}$ ) and the detection of an energetic ion beam (high energy peak corresponding to the ion beam at $V_{\text {beam }}$ ) resulting from ion acceleration through the potential drop of the double layer at $z=25 \mathrm{~cm}$.

The parameters $V_{\text {chamber }}$ and $V_{\text {beam }}$, as well as the potential drop $\phi_{D L} \sim\left(V_{\text {beam }}-V_{\text {chamber }}\right)$ of the DL are shown by open squares, triangles, and diamonds, respectively, in Fig. 2 as a function of pressure. $V_{\text {beam }}$ follows $V_{\text {source }}$ while the plasma potential $V_{\text {chamber }}$ downstream of the DL remains fairly constant at around $29 \mathrm{~V}$. The potential drop $\phi_{D L}$ of the DL also follows $V_{\text {source }}$. At $0.3 \mathrm{mTorr}, V_{\text {beam }}$ is $48 \mathrm{~V}, \phi_{D L}$ is $20 \mathrm{~V}$, and the corresponding ion beam velocity is about $10 \mathrm{~km} \mathrm{~s}^{-1}\left(v \sim \sqrt{\left(2 e \phi_{D L} / m_{i}\right.}\right)$.

Keeping the RFEA positioned at $z=37 \mathrm{~cm}$ and facing the DL, the IEDF is measured at 0.3 mTorr when decreasing the current in the solenoid near the source exit from 6 to 5,4 , and $2 \mathrm{~A}$ (Fig. 1) and the corresponding results are shown on Fig. 3. For coil current values of 6 and 5 A (solid and dotted lines, respectively), a double peak distribution is clearly observed showing the presence of the double layer. For a coil current of $4 \mathrm{~A}$ (dashed line), the distribution consists of a peak at about $23 \mathrm{~V}$ with a tail of hot ions in the $28-40 \mathrm{~V}$ range and for a coil current of $2 \mathrm{~A}$ (dotted-dashed line), a distribution with a single peak around $22 \mathrm{~V}$ is measured.

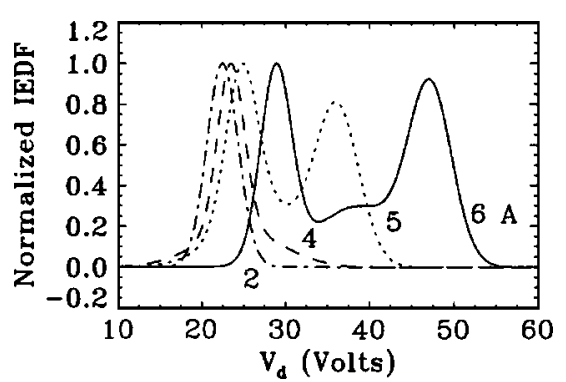

FIG. 3. Normalized IEDFs obtained with the RFEA located on axis at $z$ $=37 \mathrm{~cm}$ and facing the source for the four magnetic field cases shown in Fig. 1: coil current near the closed end of the source of $6 \mathrm{~A}$ (solid line), $5 \mathrm{~A}$ (dotted line), $4 \mathrm{~A}$ (dashed line), and $2 \mathrm{~A}$ (dotted-dashed line). The rf power and gas pressure are $250 \mathrm{~W}$ and 0.3 mTorr, respectively. 


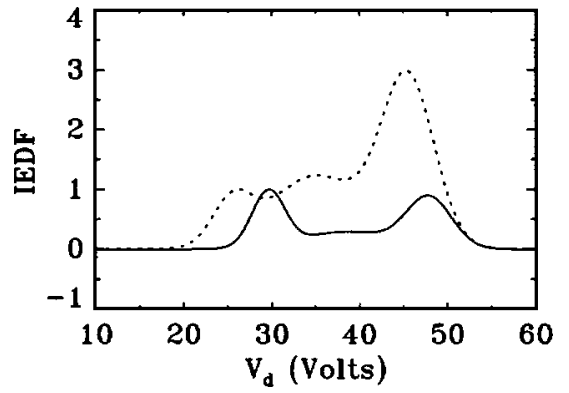

FIG. 4. IEDFS obtained with the RFEA located on axis at $z=37 \mathrm{~cm}$ and facing the source without (solid line) and with (dotted line) the addition of a second glass plate at $z=2 \mathrm{~cm}$. For better clarity, the IEDF is normalized using the low energy peak corresponding to the downstream plasma potential. The rf power and gas pressure are $250 \mathrm{~W}$ and $0.3 \mathrm{mTorr}$, respectively, and the maximum field condition shown by a solid line on Fig. 1 is used.

Measurements in the absence of magnetic field in the helicon source show a single peak IEDF at around $46 \mathrm{~V}$ and no double layer.

An additional experiment is performed by inserting a second glass plate in the source. This insulating plate can be moved from $z=0$ to $16 \mathrm{~cm}$, while operating at 0.3 mTorr and $250 \mathrm{~W}$ pressure and $\mathrm{rf}$ power conditions, respectively, and with the maximum field case shown in Fig. 1. The most striking result was obtained for $z \sim 2 \mathrm{~cm}$, which also corresponds to the position of maximum magnetic field near the closed end of the source: Fig. 4 shows the IEDF obtained with (dotted line) and without (solid line) the second glass plate. To emphazise the main outcome of this experiment, both IEDFs have been normalized at the low energy peak ( $V_{\text {chamber }} \sim 29 \mathrm{~V}$ ). The amplitude of the beam drastically increases while the DL potential drop $\left(V_{\text {beam }}-V_{\text {chamber }}\right)$ remains constant $(\sim 20 \mathrm{~V})$. The ratio between the beam density and the downstream density, determined using a deconvolution method previously described, ${ }^{12}$ is increased from 0.15 to 0.5 .

One of the main aspects of DLs is that they are the source of particle acceleration. Electron or ion beams in space plasmas have been measured using diagnostics on satellites. ${ }^{3}$ Laboratory experiments are a great complement to satellite observations, the latter being a difficult, costly and long-term task, and spatially and temporaly limited. Although laboratory plasmas differ from space plasmas in many ways, in the present context of current-free electric double layers, direct measurements of the potentials upstream and downstream of the double layer can be made in addition to the beam observations. In our system, no current or potential is imposed on the plasma as an initial condition and the potential structure of the current-free DL is selfconsistently created by the expanding plasma.

For pressures below $\sim 2 m$ Torr, high potentials ( $V_{\text {source }}$ $\sim 40-80 \mathrm{~V}$ ) are measured in the middle of the helicon source and an energetic ion beam is detected in the diffusion chamber, an indirect evidence of the presence of an electric double layer in the expansion region near the source exit. The beam energy and the potential drop of the DL are deduced from the measured IEDFs and Fig. 2 shows that $V_{\text {source }}, \phi_{D L}$, and $V_{\text {beam }}$ decrease by the same amount with increasing pressure, probably as a result of enhanced collisionality within the plasma which reduces the electron temperature. ${ }^{19}$ For pressures above 1 mTorr, the mean free path for ion-neutral collisions (charge exchange and elastic collisions) is a few $\mathrm{cm}$ which is a few times less than the distance of $12 \mathrm{~cm}$ between the DL and the RFEA and only a tail of hot ions (weakening with increasing pressure) is observed on the IEDF. The DL may still exist and may also shift axially but any beam formed while traversing the DL will have its energy transferred to neutrals over a distance of a few mean free paths. ${ }^{12}$ Above a few mTorr, collision processes will lead to an ambipolar diffusion of the plasma from the source to the chamber, well described ${ }^{10}$ by the Boltzmann relation ( $n$ $\left.=n_{0} \exp \left(e \phi / k T_{e}\right)\right)$.

The results of Fig. 3 suggest that the presence of the DL and its amplitude strongly depends on the magnetic field. No DL is observed when no magnetic field is applied and with the present operating conditions of $250 \mathrm{~W}, 0.3 \mathrm{mTorr}$, and about $135 \mathrm{G}$ near the open exit of the source, a minimum field of about $125 \mathrm{G}$ (coil current of $5 \mathrm{~A}$ ) near the closed end of the source is necessary for the DL to exist. For a coil current of $4 \mathrm{~A}(110 \mathrm{G})$, a tail of ions at energies between 30 and $45 \mathrm{~V}$ is observed in the IEDF, suggesting a high plasma potential in the source but probably no double layer: the mean free path for ion-neutral collisions (charge exchange and elastic collisions) is about $10 \mathrm{~cm}$ at a pressure of 0.3 mTorr and it is unlikely that the DL would have moved upstream by such a distance so as to prevent a beam measurement at $z=37 \mathrm{~cm}$.

Presently, the magnetic field near the source exit (and near the DL at $z=25 \mathrm{~cm}$ ) has been kept constant and the magnetic field configuration is forcing the plasma to expand from the source (insulating walls) to the diffusion chamber (conducting walls). Currents cannot flow from the source to other parts of the reactor and for the Boltzman relation to be respected along the expansion, high source potentials must exist to allow the potential drop to occur in the bulk of the plasma. At very low pressures, the collisions are limited, and the plasma can either become unstable or form a double layer near the exit of the source. The temporal stability of this DL has been recently measured ${ }^{20}$ suggesting that the plasma is able to satisfy equal losses of positively and negatively charged particles to the walls and to the diffusion chamber by setting up a current-free double layer in the expansion region defined by the geometric expansion and by the divergence of the magnetic field. The results shown in Fig. 3 suggest that the magnetic field near the closed end of the source is strongly correlated to the large source potentials.

In the present system, energy generated by an external source is fed into the plasma and is absorbed by the plasma electrons. ${ }^{19}$ No current or potential is presently imposed as a starting condition but specific boundaries, geometric and magnetic structures are present: although the position of the solenoids relative to the tube/antenna geometry is fixed in the present experimental design, it was possible to carry out a preliminary experiment by adding a second glass plate and shortening the source length. The results in Fig. 4 show that a beam density increase by a factor of 3 can be obtained with the second glass plate positioned at the maximum of the magnetic field $(z=2 \mathrm{~cm})$. This increase in the beam density 
could result from an increased plasma density upstream of the DL or from a change in the axial position of the DL since the potential drop remains fairly constant. Further experiments using a helicon source with versatile geometric settings would need to be carried out for a detailed investigation of the parameters ranges leading to the formation of this current-free electric DL.

Although there is no theory of current-free DLs available in the literature, Perkins and Sun $^{21}$ pointed out that any double-layer solution with current could be transformed into a currentless solution. Theoretical and simulation studies on collision-dominated shocks associated with propagating double layers in plasmas ${ }^{22}$ and rarefaction shocks ${ }^{23}$ may also be relevant to some aspects of this double layer. Recently, current-free DLs have been observed in particle in cell simulations of rf discharges with an inductive heating process and a loss process mimicing plasma expansion in a diverging magnetic field. ${ }^{24}$ It is very encouraging that similar currentfree DLs have now been diagnosed in four helicon systems worldwide, ${ }^{10,11,25,13}$ all with strongly divergent magnetic field structures. In addition to RFEA measurements, laser induced fluorescence measurements have been performed in some of these helicon systems which give access to flow velocities, a parameter commonly measured in space plasmas. The values are in good agreement with those derived by RFEA measurements, typically 6 to $10 \mathrm{~km} \mathrm{~s}^{-1}$ in our experiment. ${ }^{26}$ In the context of solar wind acceleration, Pike and Harrison ${ }^{27}$ have reported large flow velocities (up to $200 \mathrm{~km} \mathrm{~s}^{-1}$ ) at the foot of solar macrospicules, an event not fully understood to this date and which could also be related to acceleration caused by current-free electric double layers in a divergent magnetic field.

In summary, ion measurements using an energy analyzer have been performed upstream and downstream of a currentfree double layer as a function of pressure and magnetic field in the source. It was found that the high source potential and the energy of the ion beam generated by acceleration across the potential drop of the double layer are strongly correlated and both increase with decreasing pressure. The selfconsistent formation of the double layer by the plasma at low pressures is dependent on the magnetic field configuration and on the source geometry.

${ }^{1}$ H. Alfven and P. Carlqvist, Sol. Phys. 1, 220 (1967).

${ }^{2}$ M. A. Raadu, Phys. Rep. 178, 25 (1989), and references therein.

${ }^{3}$ L. Andersson, R. E. Ergun, D. L. Newman, J. P. McFadden, C. W. Carlson, and Y. J. Su, Phys. Plasmas 9, 3600 (2002).

${ }^{4}$ N. Sato, R. Hatekeyama, S. Iizuka, T. Mieno, K. Saeki, J. J. Rasmussen, P. Michelsen, and R. Schrittwieser, Phys. Rev. Lett. 46, 1330 (1981).

${ }^{5}$ B. H. Quon and A. Y. Wong, Phys. Rev. Lett. 37, 1393 (1976).

${ }^{6}$ G. Hairapetian and R. L. Stenzel, Phys. Rev. Lett. B3, 899 (1990).

${ }^{7}$ G. Hairapetian and R. L. Stenzel, Phys. Fluids A 65, 175 (1991).

${ }^{8}$ J. G. Andrews and J. E. Allen, Proc. R. Soc. London A320, 459 (1971).

${ }^{9}$ S. A. Andersen, V. O. Jensen, P. Nielsen, and N. D. Angelo, Phys. Fluids 12, 557 (1969).

${ }^{10}$ C. Charles and R. W. Boswell, Appl. Phys. Lett. 82, 1356 (2003).

${ }^{11}$ S. A. Cohen, N. S. Siefert, S. Stange, R. F. Boivin, E. E. Scime, and F. M. Levinton, Phys. Plasmas 10, 2593 (2003).

${ }^{12}$ C. Charles and R. W. Boswell, Phys. Plasmas 11, 1706 (2004).

${ }^{13}$ X. Sun, C. Biloiu, R. Hardin, and E. E. Scime, Plasma Sources Sci. Technol. 13, 359 (2004).

${ }^{14}$ T. Nakano, N. Sadeghi, D. J. Trevor, R. A. Gottscho, and R. W. Boswell, J. Appl. Phys. 72, 3384 (1992).

${ }^{15}$ M. A. Raadu, Space Sci. Rev. 68, 29 (1994).

${ }^{16}$ D. L. Newman, M. V. Goldman, R. E. Ergun, and A. Mangeney, Phys. Rev. Lett. 87, 255001-1 (2001).

${ }^{17}$ P. Coakley and N. Hershkowitz, Phys. Fluids 22, 1171 (1979).

${ }^{18}$ C. Charles, A. W. Degeling, T. E. Sheridan, J. H. Harris, M. A. Lieberman, and R. W. Boswell, Phys. Plasmas 7, 5232 (2000).

${ }^{19}$ M. A. Lieberman and A. J. Lichtenberg, Principles of Plasma Discharges and Materials Processing (Wiley, New York, 1994), Chaps. 10 and 12.

${ }^{20}$ C. Charles and R. W. Boswell, Phys. Plasmas 11, 3808 (2004).

${ }^{21}$ F. W. Perkins and Y. C. Sun, Phys. Rev. Lett. 46, 115 (1981).

${ }^{22}$ S. Torven, Phys. Rev. Lett. 47, 1053 (1981).

${ }^{23}$ N. Singh and G. Khazanov, J. Geophys. Res. 108, 8007 (2003).

${ }^{24}$ A. Meige, R. W. Boswell, C. Charles, J. P. Boeuf, G. Hagelaar, and M. M. Turner, IEEE Trans. Plasma Sci. (to be published).

${ }^{25}$ N. Plihon, O. Sutherland, C. Charles, R. W. Boswell, and P. Chabert (private communication).

${ }^{26}$ X. Sun, A. M. Keesee, C. Biloiu, E. E. Scime, A. Meige, C. Charles, R. Boswell, and S. Cohen, Phys. Rev. Lett. (submitted).

${ }^{27}$ C. D. Pike and R. A. Harrison, Sol. Phys. 175, 457 (1997). 